\title{
New Treatment Options in ER-Positive, HER2-Positive and Triple-Negative Breast Cancer
}

\author{
Chair: Günther Steger \\ Participants: Richard Bell ${ }^{b}$ David Cameron ${ }^{c} \quad$ Rebecca A. Dent ${ }^{d} \quad$ Christian Jackisch $^{\mathrm{e}}$ \\ aDepartment of Medical Oncology, Medical University Vienna, Austria, ${ }^{\mathrm{b} T}$ The Andrew Love Cancer Centre, The Geelong Hospital and \\ Deakin University, Geelong, Australia, 'Edinburgh Cancer Research UK Centre, The University of Edinburgh, UK, 'National Cancer Center \\ Singapore, Duke-NUS Graduate Medical School, Singapore, e'Department of Obstetrics and Gynecology, Klinikum Offenbach, Germany
}

\section{Question 1: In 2012 Several Drugs and New Treatment Options Became Available for Advanced Breast Cancer and Some More Are Expected for 2013. Which Therapy Options Have the Potential to be Immediately Incorporated Clinical Routine in ER-Positive Disease?}

Richard Bell: At several points in time over the last three decades there has been a sense that the endocrine aspects of breast cancer, with over a century of research, were a done deal. Interventions were well described and guidelines for use established; there was a feeling of satisfaction with endocrine targeted strategies with useful efficacy and limited toxicity. The challenges of breast cancer were seen to lie elsewhere. We now have proof of principle data confirming that it is possible to treat endocrine resistance. The m-TOR inhibitor everolimus has proven potential to 'reverse' or overcome endocrine resistant states. We do not yet have a clear picture regarding activity in de novo vs. acquired resistance states, and indeed struggle somewhat to define these clinically, particularly in the adjuvant (and postadjuvant) settings. The data on the CDK 4/6 inhibitor PD0332991 add complexity; this randomised phase II study included patients who relapsed early, often used as a marker of resistance. We face complex issues in selection of strategies for further development of these agents. Progress could be greatly accelerated if we can develop predictive molecular signatures associated with response, TSC1 is a possible candidate. Here, we would define and target resistance based on its mechanism. Without these markers we will blunder about, and may embark on futile studies with attendant costs and toxicity. In the fullness of time the role of these agents - beyond the current metastatic trials entry criterion - might include the utility of these agents in preventing emergence of resistance, in the treatment of primary resistance (if we can find markers of this state) and potentially in high-risk adjuvant applications.

\begin{tabular}{ll}
\hline KARGER & @ 2013 S. Karger GmbH, Freiburg \\
Fax +49 7614520714 & Accessible online at: \\
$\begin{array}{l}\text { Information@Karger.com } \\
\text { www.karger.com }\end{array}$ & www.karger.com/brc \\
&
\end{tabular}

In addition to strong translational science components, both the cost (health economics) and toxicity of these agents must be considered in developing strategies to define and refine the use of these agents. With chronic therapies the impact of toxicities on compliance and quality of life must be priorities in study design. The short patent life of everolimus may impact on resources for development of this agent.

The ATLAS study provides additional information in favour of extended duration adjuvant endocrine therapy. We will need to develop algorithms and guidelines about threshold risks for clinical recommendations on deployment of such therapy. Hopefully molecular prognosticators will in the future prove helpful here. We must consider how we manage the decade of therapy, assure compliance, and deal with the attendant economic, logistic and workforce issues - both the patient and the clinician age by a decade. Fulvestrant at $500 \mathrm{mg}$ appears a useful option in the niche clinical setting of repeated endocrine responsive metastatic disease, but this may remain a small boutique therapy in the light of the emerging data on other agents.

David Cameron: Everolimus definitely has the potential. The main challenges are going to be cost and toxicity: I suspect that we will not add everolimus to exemestane for every patient, particularly in elderly patients and those with highly endocrine sensitive disease. However, it is the first real breakthrough for postmenopausal patients with estrogen receptor-positive $(\mathrm{ER}+)$ metastatic breast cancer in about 10 years!

Rebecca Alexandra Dent: For the first time it has been shown that an mTOR inhibitor, everolimus, can overcome endocrine resistance among patients with hormone receptor-positive metastatic breast cancer. This is a huge development allowing patients to delay having to start chemotherapy. We need to examine more thoroughly the biomarker analysis from the

Univ. Prof. Dr. Günther Steger

Klinische Abteilung für Onkologie

Univ--Klinik für Innere Medizin I

Medizinische Universität Wien

Währinger Gürtel 18-20, 1090 Wien, Austria

guenther.steger@meduniwien.ac.at 
BOLERO studies however and correlate gene changes (TSC1) with response. Limitations of this approach include the metabolic syndrome in obese postmenopausal women and ensuring a good understanding of toxicity management, including appropriate dose reductions and delays when needed. A number of other approaches to reverse hormone resistance are currently being evaluated and are also being evaluated in the HER2-positive resistant disease setting.

Fulvestrant at the $500 \mathrm{mg}$ dose is another option for ER+ metastatic breast cancer. Previous studies have likely underestimated its efficacy due to the use of the lower $250 \mathrm{mg}$ dose. What to watch out for? The recent data on the CDK 4/6 (cyclin-dependent kinase 4 and 6) inhibitor PD 0332991 (Pfizer/ Onyx), which is designed to shut down cell division, are very exciting. The results from the randomized phase II study combining PD 0332991 with letrozole showed impressive 26.1 months in progression free survival (PFS) compared with letrozole alone at 7.5 months. A large number of patients in this trial had relapsed within 12 months of their adjuvant therapy which may partly explain the short PFS in the letrozole alone arm but could identify the importance of PD 0332991 in more aggressive and potentially resistant ER+ disease. PD 0332991 is an oral agent that facilitates ease of administration however it does come at some cost with an increase in hematologic toxicity mostly in the form of neutropenia, but interestingly no cases of febrile neutropenia. A phase III study is underway and results are anxiously awaited.

Christian Jackisch: In any setting of breast cancer endocrine therapy is one of the most promising treatment options as we have the combination of the predictive and prognostic impact of the ER status at hand. We have two new options at hand: one is the reassuring data of the survival benefit using fulvestrant at the dose of $500 \mathrm{mg}$. This update has been convincingly presented by Angelo di Leo at the SABCS 2012. In this context the only limiting issue is the acquired endocrine resistance, which in most of the cases leads the management into the field of chemotherapy. The publication of the Tamrad trial as well as the BOLERO trial has shed some light on a new treatment option to overcome endocrine resistance using exemestane in combination with afinitor in this subset of patients. The data demonstrate that this option works in heavily pretreated patients as well as in patients presenting not only with low-risk metatastatic involvement.

\section{Question 2: Do You See New Therapy Options in HER2-Positive Disease?}

Richard Bell: Here we have an abundance of riches. The challenge is to sort out the optimal strategies for their use. It would now appear evident that the neoadjuvant setting can provide reliable predictions of the activity of agents and combinations of agents in the HER2 positive setting. While not always reporting ahead of evaluation of similar strategies in the adjuvant and metastatic setting, these have correctly ranked the activity of trastuzumab vs. lapatinib (the discontinued arm of ALTTO), and pertuzumab plus trastuzumab vs. trastuzumab in the metastatic setting. Time will tell us if the adjuvant trastuzumab and lapatinib combination arm of ALTTO is superior, as predicted by neoALTTO, and if the combination arm of APHINITY is superior, as predicted by NEO-SPHERE.

Clearly trastuzumab and pertuzumab have exciting activity in first line therapy of metastatic disease, without any great toxicity burden. Also exciting is the activity of T-DM1 in the metastatic setting. What would the outcome of a T-DM1 plus pertuzumab combination be? Will antibody plus lapatinib combinations with promising activity, albeit with troublesome toxicity, be further evaluated? Will pharma companies engage in such cross company studies beyond ALTTO? We should utilise the neoadjuvant setting to pick potential winning strategies, and to rank therapies, with about one log less patients and with early endpoints. Without such strategies we may waste time, effort and resources in futile endeavours like the lapatinib arm of ALTTO. We should maintain a strong correlative basic science component in these studies, although it must be acknowledged that beyond HER2 itself, no predictive marker has yet any clinical utility. We are at great risk of developing effective, but unaffordable therapies. Clinicians must engage in these issues as we go forward.

David Cameron: Pertuzumab in first line and then T-DM1 are new options in HER2-positive disease. The major challenge for T-DM1 will be cost - but both these drugs have the potential to make a real difference for women with HER2-positive metastatic breast cancer. Pertuzumab in terms of increased efficacy - T-DM1 may not necessarily give better activity as a single agent, but might be a much better tolerated treatment.

Rebecca Alexandra Dent: The confirmation of benefit of pertuzumab in combination with trastuzumab for first line treatment of HER2-positive metastatic breast cancer was likely the most important new treatment. It affirmed that dual blockage was associated with improved PFS and overall survival. The updated overall survival results presented at San Antonio by Sandy Swain were impressive with median survival still not even reached for the pertuzumab treated patients. Most importantly for patients, dual blockade was associated with little increase in toxicity. The EMILIA trial highlighted the efficacy of T-DM1 showing significant improvements in PFS at ASCO and confirmed a benefit in overall survival at ESMO presented by Sunil Verma and now published in the New England Journal of Medicine. The challenge will be to decide the best sequencing of these drugs in the metastatic setting and to identify which patients could delay initiating chemotherapy. T-DM1, an immunoconjugate, represents a revolutionary change in how we deliver chemo- 
therapy. The issue for both of these agents however will be financial cost to patients and governments in a time of global fiscal constraint.

Christian Jackisch: The field of HER2 targeting treatment options promises a bright future even for those patients who present with HER2-positive disease and who are already pretreated with trastuzumab either in the neoadjuvant or adjuvant setting. Pertuzumab and trastuzumab in combination with docetaxel is a convincing alternative for these patients as well as the introduction of T-DM1 as single-drug option. Unfortunately the translational subprojects of the Cleopatra trial were unable to identify biomarkers to predict effectiveness and guide treatment decisions. In addition to this the dual blockade could also be extended to the combination of trastuzumab and lapatinib in the later line of metastatic breast cancer treatment or might be an option for those patients not accepting or tolerating additional chemotherapy in this setting.

\section{Question 3: In Your Opinion, What Are New Options in Triple-Negative Disease?}

Richard Bell: We have witnessed a near fiasco, with triplenegative disease being used a surrogate for basal and 'basal like' breast cancer, the evaluation of PARPI and putative PARPI agents as well as of DNA damaging chemotherapy regimens. No clinically useful information or therapies resulted. We now know that this subset is not one subset, but a heterogeneous group of ER-, PR- and HER2-negative diseases. The BEATRICE study has told us that antiangiogenic therapy is ineffective in these patients, but importantly shows that with modern adjuvant therapies the outlook is much better than suggested by past, mostly retrospective series.

What is the way forward? I believe that it will be with neoadjuvant studies, with strong molecular components, including the study of residual disease. These would allow for the potential of adaptive designs, based on molecularly identified targets - truly personalised medicine. This will necessarily subdivide an already small group of patients, and such studies will require large and active collaborations.

David Cameron: At the moment there are no new options for these patients. It remains a challenging area - I suspect that the progress will come when we start to dissect this disease into different subgroups: perhaps for the BRCA1/2 genotype patients PARP inhibitors will prove their value, but am not sure that this will happen in 2013.

Rebecca Alexandra Dent: The biggest news for triple-negative breast cancer in 2012 is that numerous investigators have confirmed that it is a heterogeneous disease that is composed of several distinct subtypes. The Nature publication by Matt Ellis et al. from the Cancer Genomic Atlas Network also identified distinct mutations that could be used to identify targets for therapy in the future. More recently Balko and colleagues from Vanderbilt University highlighted at SABCS 2012 that in patients with triple-negative breast cancer with residual disease after neoadjuvant chemotherapy, Next Generation Sequencing identified that approximately $90 \%$ of patients had aberrations in one or more targetable pathways (i.e., DNA repair, PI3K/mTOR, RAF/MEK, cell cycle/mitotic spindle inhibitors and targeted receptor tyrosine kinases). The only such potential agent that is currently licensed is the chemotherapy eribulin through its effects on spindle microtubule dynamics. At San Antonio this year, the phase III trial failed to demonstrate statistically significant superiority of eribulin over capecitabine. However, eribulin did demonstrate a nonsignificant trend toward better overall survival, particularly in patients with triple negative disease (14.4 vs. 9.4 months, hazard ratio 0.70 ). Thus, much like other agents for advanced breast cancer, there may be certain patient subgroups with greater therapeutic benefit. We need to design smarter adaptive trials that are personalized to tailor therapy to a patient's tumor's given biology.

Christian Jackisch: Treatment of triple-negative breast cancer remains a hopeless field of new drugs at the moment, as it is still uncertain whether the PARP inhibitors still are to come. In some cases the addition of bevacizumab might be an option in the metastatic setting. No specific targeted drug or chemotherapy combination has been identified at the moment to be able to take the lead. Without any question this is the field of unmet medical needs: searching for robust data to improve the management of triple-negative breast cancer.

\section{Participants}

Richard Bell, MD

The Andrew Love Cancer Centre

The Geelong Hospital and Deakin University

70 Swanston Street, Geelong 3250, Australia

rbell@pipeline.com.au

David Cameron, MD

Edinburgh Cancer Research UK Centre

The University of Edinburgh

Crewe Road South, Edinburgh EH4 2XR, UK

David.Cameron@igmm.ed.ac.uk

Rebecca A. Dent, MD

National Cancer Center Singapore

Duke-NUS Graduate Medical School Singapore

8 College Road

Singapore 169857, Singapore

rebecca.dent@duke-nus.edu.sg

Prof. Dr. med. Christian Jackisch

Klinik für Gynäkologie und Geburtshilfe

Klinikum Offenbach $\mathrm{GmbH}$

Starkenburgring 66, 63069 Offenbach, Germany

Christian.Jackisch@klinikum-offenbach.de 